\title{
Lyophilisate and Solvent for Emulsion for Injection
}

National Cancer Institute

\section{Source}

National Cancer Institute. Lyophilisate and Solvent for Emulsion for Injection. NCI

Thesaurus. Code C158597.

Sterile lyophilisate (freeze-dried powder) and sterile solvent intended for the preparation of an emulsion for injection, for veterinary use, by mixing the powder and the solvent. 